\title{
Application of Improved Interval Numbers TOPSIS Method Based on Mahalanobis Distance in the Evaluation of SME Public Service Platform
}

\author{
Wenyan Dong and Xinzhong Bao* \\ Management College, Beijing Union University, Beijing, China, 100101 \\ ${ }^{*}$ Corresponding author
}

\section{基于马氏距离改进 TOPSIS 方法的中小企业公共服 务平台评价}

\author{
董文妍, 鲍新中* \\ 北京联合大学管理学院, 北京, 中国, 100101 \\ *通讯作者
}

\begin{abstract}
SME public service platform is an economic organization providing a wide range of common services for small and medium-sized enterprise group. It is designed to help SMEs to transform the development mode, to realize the optimal allocation of resources, and to reduce their survival and development cost. This paper aims at the evaluation issue of SME public service platform. 21 specific evaluation indexes of two levels and seven aspects are put forward. The interval number TOPSIS method based on Mahalanobis distance recommended. The use of interval value makes the assignment of qualitative indexes more reasonable. The replacement for Mahalanobis distance eliminates the influence of the linear correlation between the attributes when using Euclidean distance. Finally, an example is used to test that this new method is more scientific and reasonable.
\end{abstract}

Keywords-SME public service platform; interval number; mahalanobis distance; TOPSIS method

摘要一中小企业公共服务平台是为中小企业群体提供各类共性 服务的经济组织, 旨在帮助企业转变经济发展方式, 实现资源优 化配置, 降低其生存和发展成本。本文针对中小企业公共服务平 台的考核评价方法问题进行研究，通过两个层面 7 个方面 21 项 具体的评价指标, 运用基于马氏距离的区间数 TOPSIS 方法对中 小企业公共服务平台进行考核评价。区间值的运用使得难以进 行赋值的定性指标得以较为合理的体现, 并且运用马氏距离对 欧氏距离进行替换，克服了属性之间存在线性相关性时欧氏距 离失效的缺陷, 使该方法更具科学性和合理性, 最后通过中小 企业公共服务平台考核评价实例对该方法进行检验。
关键词一中小企业公共服务平台; 区间数; 马氏距离; TOPSIS 方法

\section{I. 引言}

当前经济体制下，中小企业在国民经济发展、解决社会 就业问题中发挥着越来越重要的作用, 而中小企业创新能 力不足成为制约其发展的重要因素，所以，搭建中小企业 公共服务平台，帮助企业提升其技术创新能力成为各地政 府致力推进的一个重要事项，因此，政府都会通过资金投 入、政策支持等来完善当地中小企业服务平台的建设。学 者们也围绕中小企业服务平台建设问题展开了一些理论研 究, 主要包括中小企业服务平台的建设问题 ${ }^{[1-2]}$ 、公共服务 体系构建 ${ }^{[3]}$ 、商业模式 ${ }^{[4]}$ 或盈利模式 ${ }^{[5]}$ 的构建、平台运营情 况分析 ${ }^{[6-7]}$ 等方面。为加强对中小企业公共服务平台的规范 管理, 逐步形成服务平台自我完善、专项考核和社会监督 相互促进的评价模式, 实现平台的示范带动作用, 确保服 务平台实现可持续发展, 需要通过制定相关指标对运用适 当方法对服务平台进行考核评价。中小企业公共服务平台 考核评价实际上是一个多属性决策问题，评价方法是影响 平台考核的重要外生变量, 而国内外对于这一问题的研究 很少。本文基于区间数属性值，提出基于马氏距离的相对 距离计算方法，代替传统的欧氏距离，从而对 TOPSIS 贴 进度的计算方式进行改进, 最终以中小企业公共服务平台 考核评价为算例, 验证该方法的合理性和有效性。 


\section{II. 基于马氏距离对区间数 TOPSIS 方法的改进}

\section{A. 马氏距离的含义}

马氏距离是 1936 年由印度统计学家 Mahalanobis 提出的 一种统计距离计算方法, 因其具有不受坐标之间量纲化影 响、消除了属性之间相关性等独特优点, 被广泛应用于多 属性分析中, 假设 $\mathrm{A}_{\mathrm{i}}$ 的数据向量 $A_{t}=\left(b_{t 1} b_{t 2 *} b_{i n}\right)$ 表示 第 $\mathrm{i}$ 个样本所对应的属性坐标值, $S^{+}=\left\{s_{1}^{+}, g^{+}, \ldots, s_{n}^{+}\right\}$、 $S^{-}=\left\{s_{1}^{-}, s_{2}-s_{n}^{-}\right\}$分别表示正、负理想解所对应的空间 坐标。当给定原始数据后, 由于 $\mathrm{A}_{\mathrm{i}}$ 的坐标是变化的, 因此 设 $\delta_{1}, \delta_{2} \ldots \delta_{n}$ 表示 $\mathrm{n}$ 个属性变量 $b_{2}, b_{2}=b_{n}$ 的 $\mathrm{m}$ 次观测的 样本方差。则第 $\mathrm{i}$ 个方案 $\mathrm{A}_{\mathrm{i}}$ 到 $S+$ 牤 $S$-的马氏距离分别为:

$$
\begin{aligned}
& \mathrm{d}\left(A_{t} S^{+}\right)=\sqrt{\left(\left(\mathrm{b}_{t}-S^{+}\right) F^{2}\right) \Sigma^{-1}\left(\mathrm{~b}_{t}-S^{+}\right)} \\
& \mathrm{d}\left(A_{t} S^{-}\right)=\sqrt{\left(\left(\mathrm{b}_{t}-S^{-}\right) F^{2}\right) \Sigma^{-1}\left(\mathrm{~b}_{t}-S^{-}\right)}
\end{aligned}
$$

其中, $\Sigma^{-1}$ 是属性指标间的协方差逆矩阵。

由此得到各方案的相对贴进度为:

$$
c_{t}^{*}=\frac{d\left(A_{i}, S^{-}\right)}{d\left(A_{t}, S^{+}\right)+d\left(A_{t}, S^{-}\right)}
$$

\section{B. 基于区间数的马氏距离}

为了计算区间型随机变量的马氏距离, 本文给出了区间 型变量协方差及协方差矩阵的定义。

定义 1 : 设 $S 、 U$ 为区间型随机变量, $\mathrm{E}\{[S-E(S)][U-E(U)]]$ 称为随机变量 $\mathrm{S}$ 与 $\mathrm{U}$ 的协方差,

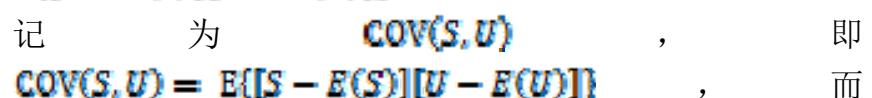

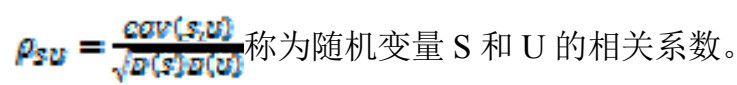

定义 2: 若 $\mathrm{n}$ 维区间型随机变量 $\left(S_{1}, S_{\Omega_{\nu 1}=}, S_{n}\right)$ 的二阶混 合 中 心 矩 $c_{t j}=\operatorname{Cov}\left(S_{t}, S_{j}\right)=E\left\{\left[S_{t}-E\left(S_{j}\right)\right] \cdot\left[S_{j}-E\left(S_{t}\right)\right]\right]$ 都 存 在, 则称矩阵

$$
C=\left[\begin{array}{cccc}
C_{11} & C_{12} & \cdots & C_{1 n} \\
C_{g 1} & C_{g 2} & \cdots & C_{g n} \\
\vdots & \vdots & C_{n} & \vdots \\
C_{n 1} & C_{n 2} & \cdots & C_{n n}
\end{array}\right]
$$

为 $\mathrm{n}$ 维区间型随机变量的协方差矩阵, 显然协方差矩阵为 对称矩阵。 为:

因此, 第 $\mathrm{i}$ 个备选方案正负理想解之间的马氏距离分别

$$
D_{t}^{+}=\mathrm{d}\left(A_{t} S^{+}\right)=\sqrt{\left[\left(\left(p_{t}-S+\right)\right)^{2} C^{-1}\left(\gamma_{t}-S+\right)\right]}
$$

$$
D_{t}^{-}=\mathrm{d}\left(A_{t} S^{-}\right)=\sqrt{\left[\left(\gamma_{t}-S^{-}\right) F^{-1} C^{-1}\left(\gamma_{t}-S^{-}\right)\right]}
$$

各评价方案的相对贴进度:

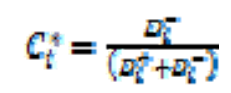

III. 中小企业公共服务平台考核评价的实证分析

\section{A. 建立评价指标体系}

结合各地对中小企业公共服务平台评价的实践，本文从 实体运营方面的三个方面: 运营管理、服务业绩和社会评 价, 以及网络运营的四个方面: 网络稳定性和安全性、网 络应用、系统第三方测评和线上服务率, 建立了中小企业 公共服务平台考核评价指标体系。其中, 在这几个方面又 细分为 21 个具体指标进行量化分析, 如表 1 所示。

\section{B. 样本描述及数据获取}

选取 8 家公共服务平台作为样本评价对象, 分别为 $\mathrm{X} 1, \mathrm{X} 2, \mathrm{X} 3, \mathrm{X} 4, \mathrm{X} 5, \mathrm{X} 6, \mathrm{X} 7, \mathrm{X} 8$, 而 U1-U21 分别表示表 1 中中 小企业公共服务平台考核评价指标体系中的 21 个具体指 标。为了评价的客观性, 本文采用对每一个指标的评分用 区间值来表示, 得到初始数据源。

\section{C. 基于离差最大化原理确定的区间数指标权重}

针对原始数据, 根据公式进行数据的规范化处理。基于 规范化决策矩阵, 利用离差最大化原理来确定各个指标属 性的权重, 计算得到中小企业公共服务平台考核评价指标 体系各个指标的权重。利用属性的权重向量 $\omega$ 和规范化后的 决策矩阵 $\widetilde{\mathrm{R}}$, 构造加权规范化决策矩阵 $\widetilde{Z}$ 。根据加权规范化 后的数据, 利用下面两式确定区间型正、负理想点。

\section{D. 改进的TOPSIS 方法计算过程}

利用传统的 TOPSIS 方法下各方案的相对贴进度计算结 果。然后, 运用 Eviews 求解各属性指标之间的相关系数矩 阵。从结果可以看出, 某些指标之间存在着较为显著的相 关性, 例如 U8 和 U15 之间的相关系数为 $-0.8836, \mathrm{U} 3$ 和 U8 之间的相关系数也达到了 -0.8608 , 表明这些指标之间存 在较高的相关性。在这种情况下, 欧氏距离失效, 所以使 用马氏距离进行计算可以使结果更加合理。运用 Matlab 软 件求得各公共服务平台到正、负理想解的马氏距离及相对 贴进度。

\section{E. 评价结果与对比分析}

根据前文的计算与分析, 得到两种方法的排序结果如表 2 所示。可以看出, 应用马氏距离改进的 TOPSIS 方法所得 到的考核评价结果与传统 TOPSIS 方法得到的结果存在很 大差异, 这是因为各属性指标之间存在较大的相关性, 传 统的 TOPSIS 方法中所使用的欧氏距离失效, 而马氏距离具 有不受变量之间相关性影响的优良特征, 可以很好地解决 传统 TOPSIS 方法的这一缺陷, 使得排序结果更加科学合 理, 也进一步证明了本文所提出的运用区间型属性值基于 马氏距离改进的 TOPSIS 方法在中小企业公共服务平台的 考核评价中更具有效性。 
表 I 中小企业公共服务平台考核评价指标体系

\begin{tabular}{|c|c|c|c|}
\hline 一级指标 & 二级指标 & 三级指标 & 代码 \\
\hline \multirow{15}{*}{ 实体运营 } & \multirow{3}{*}{ 运营管理 } & 内部管理服务制度、相关服务支撑体系的完善程度 & U1 \\
\hline & & 相应团队建设的是否能保障平台的正常运行和企业服务的正常开展 & $\mathrm{U} 2$ \\
\hline & & 平台网络自我完善和发展的能力是否良好 & $\mathrm{U} 3$ \\
\hline & \multirow{9}{*}{ 服务业绩 } & 协助中小企业举办各种会展、论坛、宣传的次数 & $\mathrm{U} 4$ \\
\hline & & 促成企业与各类金融机构的融资合作的金额数 & U5 \\
\hline & & 为企业提供各类管理咨询服务的次数, 如培训服务、战略指导、财税咨询等 & U6 \\
\hline & & 为企业推荐或直接派遣科技人才的次数 & $\mathrm{U} 7$ \\
\hline & & 为企业提供专业技术及知识产权相关服务的次数, 如技术研发、专利申报等 & U8 \\
\hline & & 是否能为企业提供全面、及时的政策解读和咨询服务 & U9 \\
\hline & & 是否建立平台服务企业的档案, 是否形成定期汇报制度 & $\mathrm{U} 10$ \\
\hline & & 统计分析、调查研究结果是否能为政府决策提供支持 & U11 \\
\hline & & 是否做到窗口线上平台互联互通 & U12 \\
\hline & \multirow{3}{*}{ 社会评价 } & 企业对平台服务的满意度 & U13 \\
\hline & & 平台日常检查、大型活动的完成度 & U14 \\
\hline & & 新闻媒体正面宣传报道数量 & U15 \\
\hline \multirow{6}{*}{ 网络运营 } & $\begin{array}{c}\text { 网络稳定性 } \\
\text { 安全性 }\end{array}$ & 是否能及时处理网络稳定性、安全性方面的问题 & U16 \\
\hline & \multirow{3}{*}{ 网络应用 } & 网站注册企业数量 & U17 \\
\hline & & 平台入围合作机构服务数量 & U18 \\
\hline & & 日均访问量 & U19 \\
\hline & $\begin{array}{c}\text { 系统第三方 } \\
\text { 测评 } \\
\end{array}$ & 第三方测评结果 & $\mathrm{U} 20$ \\
\hline & 线上服务率 & 第三方评估结果 & $\mathrm{U} 21$ \\
\hline
\end{tabular}

表 II 中小企业公共服务平台考核评价结果

\begin{tabular}{ccccccccc}
\hline & X1 & X2 & X3 & X4 & X5 & X6 & X7 & X8 \\
\hline $\begin{array}{c}\text { 传统 TOPSIS 方法 } \\
\begin{array}{c}\text { 马氏距离改进 } \\
\text { TOPSIS 方法 }\end{array}\end{array}$ & 2 & 8 & 4 & 3 & 2 & 6 & 1 & 5 \\
\hline
\end{tabular}

\section{IV. 结论与启示}

中小企业公共服务平台通过为中小企业提供服务来满足 中小企业的需求, 具有服务开放性、公益性、有偿性、资 源共享性等特点, 已经慢慢成为中小企业生存和发展过程 中不可或缺的重要支撑因素。为了督促中小企业公共服务 平台不断完善自身, 实现服务平台的可持续发展, 需要对 平台进行多方面的考核与评价, 通过设置一定的考核评价 指标, 来实现对平台的综合评价。本文运用区间数对中小 企业公共服务平台进行考核评价, 解决了定性指标衡量困 难的问题, 对定性指标在一定区间内赋值, 使得评价更加 准确。同时采用基于马氏距离的 TOPSIS 方法, 解决了传 统 TOPSIS 法中由于属性指标之间存在相关性而使欧氏距 离失效的缺陷。综合区间型属性值、马氏距离建立的更加 全面的 TOPSIS 方法, 可以产生相对应的更加合理化的评
价结果。通过本文的实例分析, 验证了基于马氏距离的区 间数 TOPSIS 方法在对中小企业公共服务平台进行考核评 价时的可行性和有效性, 具有较好的实践意义。

\section{致谢}

本文为北京市教委市属高校创新能力提升计划项目 （PXM201601420900001800202730 FCG）和北京市属 高校高水平教师队伍建设支持计划长城学者培养计划项目 （CIT\&TCD20180327）的阶段性成果之一。

\section{REFERENCE}

[1] Wang Junfeng.Building a Public Technology Service Platform for Small and Medium-sized Enterprises-The Experience of the German Fraunhofer Association and Its Enlightenment to China[J].China Science and Technology Forum.2007,10:51-54

[2] Lu Guixing.Construction of Technology Innovation Service Platform for SMEs-Based on Weifang Survey[J].Science and technology management research.2012, 15:9-12

[3] Zhang Ziwei, Yu Liufang, Wang Lingna.Research on Public Service System to Promote the Development of Small and Medium-sized Enterprises Industry-Taking Shiyan City, Hubei Province as an Example[J].Contemporary economy.2016,12:65-69

[4] Li Wei, Wang Zhe, Chen Jieyun.Research on Business Model of SME Collaborative Manufacturing Service Platform[J].Business economics research.2015,03:101-103 
[5] Li Xianghui.Research on Profit Model of SME Innovation Service Platform Based on Value Chain[J].Scientific and technological progress and countermeasures.2009,26(11): 115-118

[6] Fu Xianfeng, Mei Qiang.Efficiency Analysis of China's SME Public Service Platform Based on DEA[J].Science and technology management research.2012,11:66-69

[7] Huang Yan, Tao Qiuyan, Hao Shuomin.Survey on the application status of SME public service platform[J].Finance and accounting monthly.2016,02:3-7

[8] JAHANSHLOO G R,LOTFI F H,LZADIKHAH M. An algorithmic method to extend TOPSIS for decision-making problems with interval date [J]. Applied mathematics and computation, 2006, 175(2): 1375-1384.

[9] Wang Erwei, Qi Yanxin, Liao Aihong. Evaluation of People's Livelihood Status in Pearl River Delta Based on Improved TOPSIS Method[J]. Practice and understanding of mathematics,2016,46(18): 63-68.

[10] Hua Xiaoyi, Tan Jingxin. TOPSIS method based on "vertical" distance orthogonal projection method[J]. System Engineering Theory and Practice, 2004,(1): 114-119. 